\title{
12/15-lipoxygenase-mediated enzymatic lipid oxidation regulates DC maturation and function
}

\author{
Tobias Rothe, ${ }^{1,2}$ Florian Gruber, ${ }^{3,4}$ Stefan Uderhardt, ${ }^{1,2}$ Natacha Ipseiz,, ${ }^{1,2}$ Susanne Rössner, ${ }^{5}$ Olga Oskolkova, ${ }^{6}$ Stephan Blüml, ${ }^{7}$ \\ Norbert Leitinger, ${ }^{8}$ Wolfgang Bicker, ${ }^{9}$ Valery N. Bochkov, ${ }^{6}$ Masayuki Yamamoto, ${ }^{10}$ Alexander Steinkasserer, ${ }^{5}$ Ceorg Schett, ${ }^{1}$ \\ Elisabeth Zinser, ${ }^{5}$ and Cerhard Krönke ${ }^{1,2}$

\begin{abstract}
'Department of Internal Medicine 3 and Institute for Clinical Immunology and ${ }^{2}$ Nikolaus Fiebiger Center of Molecular Medicine, University Hospital Erlangen, University of Erlangen-Nuremberg, Erlangen, Germany. ${ }^{3}$ Department of Dermatology and ${ }^{4}$ Christian Doppler Laboratory for Biotechnology of Skin Aging, Department of Dermatology, Medical University of Vienna, Vienna, Austria. ${ }^{5}$ Department of Immune Modulation at the Department of Dermatology, University Hospital Erlangen, Erlangen, Germany. ${ }^{6}$ Department of Vascular Biology and Thrombosis Research, Center for Physiology and Pharmacology, and 'Division of Rheumatology, Internal Medicine III, Medical University of Vienna, Vienna, Austria. ${ }^{8}$ Department of Pharmacology, University of Virginia, Charlottesville, Virginia, USA. ${ }^{9}$ FTC-Forensic-Toxicological Laboratory Ltd., Vienna, Austria. ${ }^{10}$ Department of Medical Biochemistry, Tohoku University Graduate School of Medicine, School of Medicine, Sendai, Japan.
\end{abstract}

\begin{abstract}
DCs are able to undergo rapid maturation, which subsequently allows them to initiate and orchestrate T cell-driven immune responses. DC maturation must be tightly controlled in order to avoid random $\mathrm{T}$ cell activation and development of autoimmunity. Here, we determined that 12/15-lipoxygenase-meditated (12/15-LO-mediated) enzymatic lipid oxidation regulates DC activation and fine-tunes consecutive T cell responses. Specifically, 12/15-LO activity determined the DC activation threshold via generation of phospholipid oxidation products that induced an antioxidative response dependent on the transcription factor NRF2. Deletion of the 12/15-LO-encoding gene or pharmacologic inhibition of 12/15-LO in murine or human DCs accelerated maturation and shifted the cytokine profile, thereby favoring the differentiation of Th17 cells. Exposure of 12/15-LO-deficient DCs to 12/15-LO-derived oxidized phospholipids attenuated both DC activation and the development of Th17 cells. Analysis of lymphatic tissues from 12/15-LO-deficient mice confirmed enhanced maturation of DCs as well as an increased differentiation of Th17 cells. Moreover, experimental autoimmune encephalomyelitis in mice lacking 12/15-LO resulted in an exacerbated Th17-driven autoimmune disease. Together, our data reveal that 12/15-LO controls maturation of DCs and implicate enzymatic lipid oxidation in shaping the adaptive immune response.
\end{abstract}

\section{Introduction}

DCs comprise a heterogenic group of antigen-presenting cells, which share the ability to prime naive T cells (1). Upon exposure to pathogen-associated molecular patterns and endogenous danger signals, DCs undergo a maturation process, which is a prerequisite for initiating specific T cell responses. DC maturation involves a change in the pattern of secreted cytokines as well as the upregulation of costimulatory molecules. Furthermore, DC-derived signals guide the immune response by orchestrating the differentiation of naive T cells into specific effector T cell subsets. Since DCs not only ingest pathogens but also acquire self-antigens during the steady state, their maturation has to be tightly controlled to avoid priming of autoreactive T cells and autoimmunity (2).

The enzyme 12/15-lipoxygenase (12/15-LO) represents the murine ortholog of human 15-lipoxygenase (15-LO). Both enzymes oxygenate free and esterified polyunsaturated fatty acids (3).12/15LO has been shown to be expressed in foam cells within the atherosclerotic plaque and has been implicated in the oxidation of LDL particles and generation of proinflammatory lipid oxidation products that drive vascular inflammation and the development of atherosclerotic plaques $(4,5)$. However, the physiological role of this

Authorship note: Elisabeth Zinser and Gerhard Krönke contributed equally to this work. Conflict of interest: The authors have declared that no conflict of interest exists. Submitted: August 11, 2014; Accepted: February 27, 2015.

Reference information: J Clin Invest. 2015;125(5):1944-1954. doi:10.1172/JCI78490. enzyme is still incompletely understood. During the steady state, 12/15-LO is selectively expressed in different cells of the myeloid lineage, such as resident and alternatively activated macrophages. Furthermore, 12/15-LO is robustly induced upon exposure of monocytes to Th2 cytokines, such as IL-4 and IL-13 (6). Notably, this enzyme also participates in the generation of distinct antiinflammatory lipid mediators and thereby contributes to the resolution of inflammation, the limitation of inflammation-associated tissue damage, and the clearance of apoptotic cells (7-11). Although 15-LO has been reported to be expressed in human DCs (12), a potential role of this enzyme in DCs or during the development of a specific $\mathrm{T}$ cell response has remained elusive.

In the present study, we describe a role that we believe to be novel for 12/15-LO during the regulation of DC maturation and the shaping of the consecutive $\mathrm{T}$ cell response. 12/15-LO expression in DCs and 12/15-LO-derived lipid mediators limited the maturation process of DCs via activation of the transcription factor NF-E2-related factor 2 (NRF2) and dampened the differentiation of Th17 cells. Accordingly, 12/15-LO-deficient (Alox15-/) mice displayed an increased maturation of DCs as well as an enhanced differentiation of Th17 T cells, resulting in exacerbation of experimental autoimmune encephalomyelitis (EAE).

\section{Results}

Bone marrow-derived DCs express 12/15-LO. Although 15-LO can be detected in human monocyte-derived DCs (moDCs), a functional 

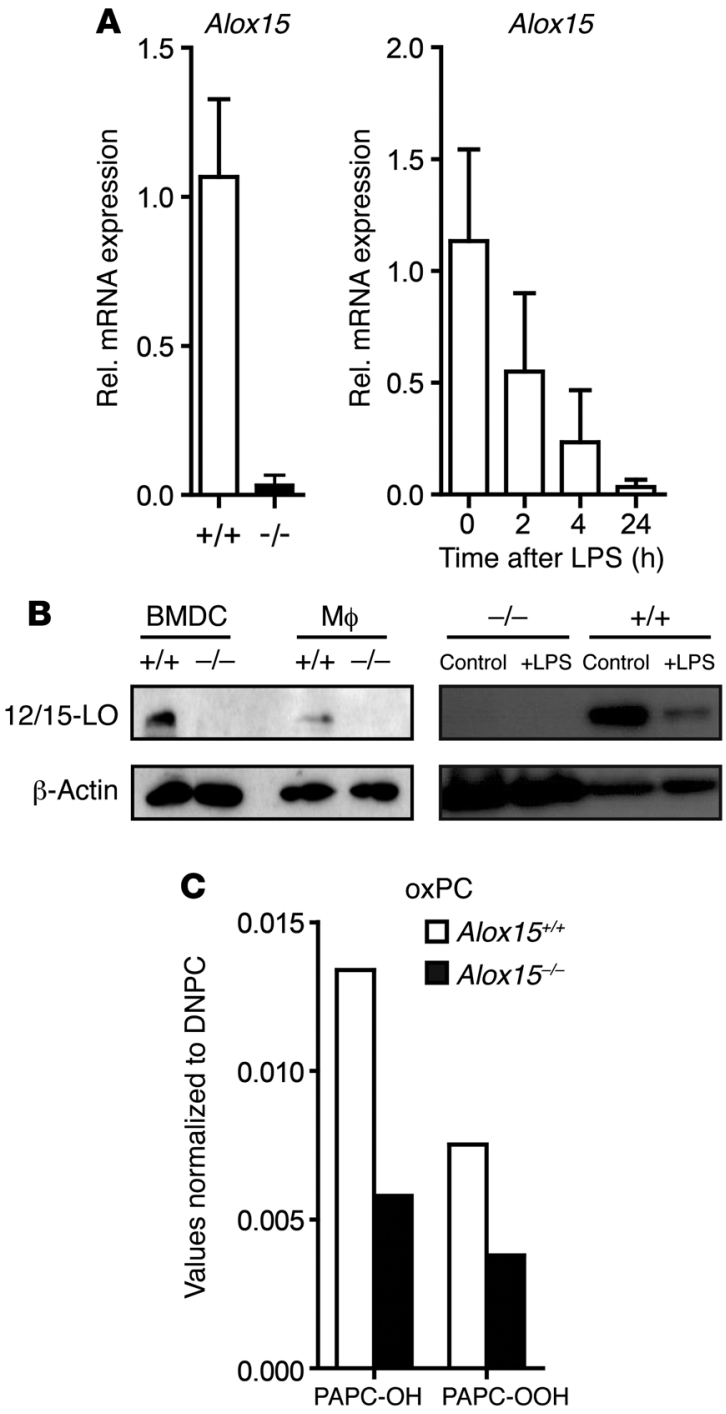

role of this enzyme in DC biology has not yet been determined (12). Notably, studies describing an oxidized LDL-mediated inhibition of DC maturation in dyslipidemic Apoe $e^{-/}$mice provide indirect evidence for a major role of lipid oxidation in DC biology (13). Therefore, we initially determined expression of 12/15-LO in murine DCs. Bone marrow-derived DCs (BM-DCs) displayed robust expression of Alox15 mRNA and of 12/15-LO protein (Figure 1, A and B). Alox15 mRNA and 12/15-LO protein expression were gradually downregulated during the DC maturation process in response to LPS (Figure 1, A and B), demonstrating that 12/15LO expression inversely correlated with the maturation status of DCs. Since membrane phospholipids represent a major substrate of this enzyme (14), we screened for the presence of 12/15-LOderived phospholipid oxidation products in DCs by applying mass spectrometry. DCs from WT mice were enriched in 12/15-LOderived oxidation products of phosphatidylcholine (PAPC-OH and PAPC-OOH), while their accumulation was reduced in DCs from Alox $15^{-/-}$mice (Figure 1C).

12/15-LO regulates the maturation process of murine and human DCs. To elucidate the role of 12/15-LO in DC function, we studied the phenotype of WT and Alox $15^{-/}$DCs before and
Figure 1. BM-DCs express functionally active 12/15-LO. (A) Quantification of Alox15 mRNA expression levels in immature BM-DCs from WT and Alox $15^{-/-}$mice or of Alox15 mRNA expression in WT BM-DCs after stimulation with LPS $(100 \mathrm{ng} / \mathrm{ml})$ for indicated intervals. Error bars represent SEM. (B) Western blot analysis of 12/15-LO protein levels in extracts of BM-DCs and peritoneal macrophages $(\mathrm{M} \Phi)$ isolated from WT and Alox15 mice and WT or Alox15 $-1-$ BM-DCs after maturation with LPS $(100 \mathrm{ng} / \mathrm{ml}$ for 24 hours). (C) Determination of different 12/15-LO-derived oxidation products in WT and Alox $15^{-1-}$ BM-DCs, as quantified by mass spectrometry. Peak area was normalized to an internal standard (1,2-dinonanoylsn-glycero-3-phosphocholine [DNPC]). Data shown are representative of 3 independent experiments $(n=3)$.

after LPS-induced maturation. Flow cytometric analysis showed that, after maturation with LPS, Alox $15^{-1-}$ DCs expressed higher levels of MHC class II molecules, costimulatory molecules, and activation markers, including CD40, CD25, CD86, CD83 and CD80, on their surfaces (Figure 2, A-C, and Supplemental Figure 1A; supplemental material available online with this article; doi:10.1172/JCI78490DS1), indicative of an enhanced maturation status. Alox15 $1-$ DCs displayed both an increase in the percentage of maturated DCs and in the expression level of the individual costimulatory molecules. Accordingly, enzymatic inhibition of 15-LO with Baicalein in human moDCs resulted in a robust upregulation of costimulatory molecules and MHC class II molecules, suggesting a spontaneous maturation of otherwise immature DCs in the absence of an enzymatically active 15-LO (Figure 2D and Supplemental Figure 1B). Together, these data revealed an important role for murine 12/15-LO and human 15-LO in controlling the activation threshold and maturation process of DCs.

12/15-LO-derived phospholipid oxidation products attenuate DC maturation. 12/15-LO catalyses oxidation of free fatty acids or of fatty acids esterified to phospholipids (11). To identify the 12/15-LO enzymatic products responsible for the inhibitory effect on DC maturation, we studied the effects of different known 12/15-LO-derived lipid mediators on this process. The 12/15-LOderived eicosanoids 12-HETE, 15-HETE, 13-HODE, and lipoxin $\mathrm{A}_{4}$ had no significant effect on LPS-induced DC maturation (data not shown). In contrast, oxidized phosphatidylcholine (oxPC) potently inhibited the maturation process of DCs and blocked the upregulation of costimulatory molecules and of activation markers, such as MHC class II molecules, CD40, CD25, CD86, CD83, and CD80 (Figure 3 and Supplemental Figure 2). Notably, oxPC treatment attenuated DC maturation and corrected the hypermaturated phenotype of Alox $15^{-/}$DCs. These findings suggested that $12 / 15-\mathrm{LO}$-derived phospholipid oxidation products counteracted the LPS-induced maturation process and thereby stabilized DCs in a resting state.

12/15-LO-derived oxPC modulates DC maturation via activation of Nrf2. Oxidized phospholipids have been previously shown to induce an antioxidative response program via the transcription factor NRF2 (15). To address the mechanisms underlying oxPCmediated inhibition of DC maturation, we investigated a potential role of NRF2. Upon activation, NRF2 was shown to enter the nucleus, bind to its responsive elements, and drive transcription of a set of genes involved in the antioxidative response, such as heme oxygenase-1 (Hmox1), quinone oxidoreductase 1 (Nqo1), or 
A

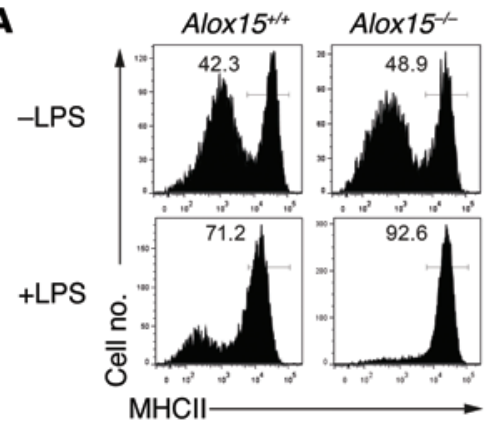

B
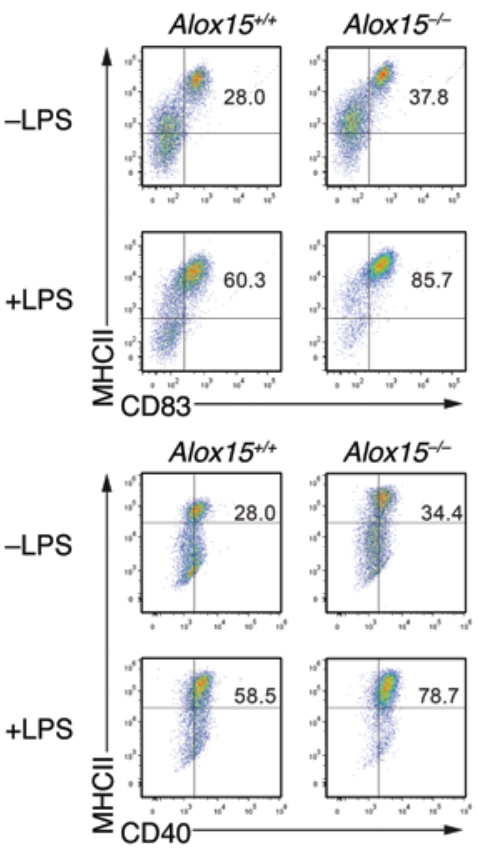
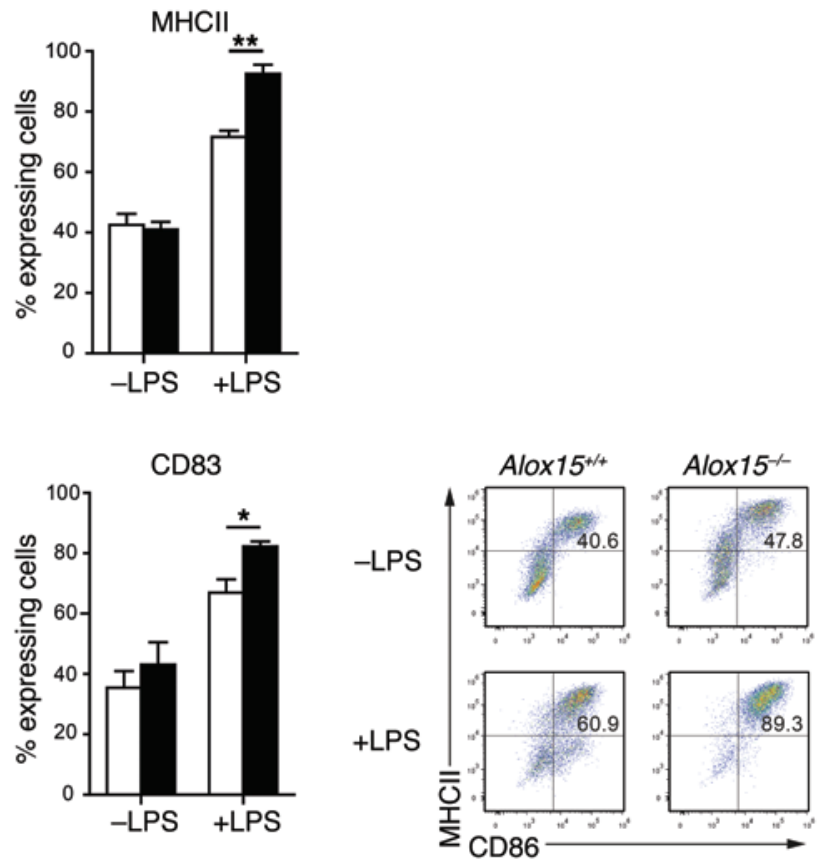

CD40

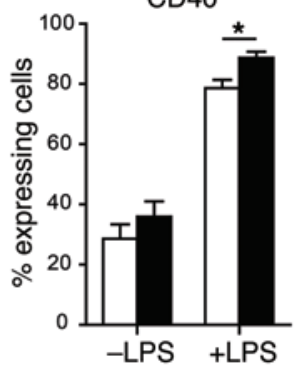

CD86

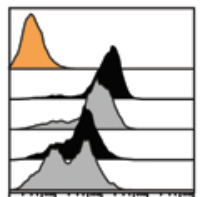

CD86

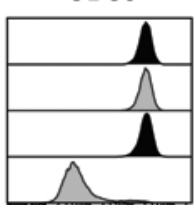

口Alox $15^{+/+}$

- Alox $15^{-1}$

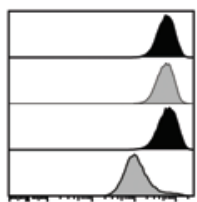

CD83

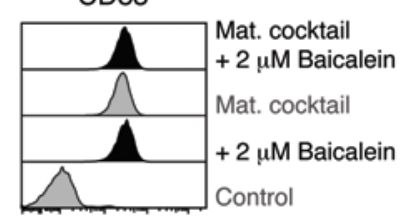

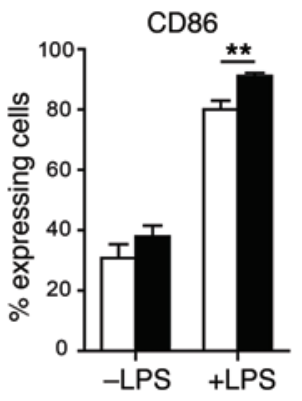

C

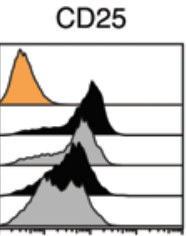

CD80

Murine BM-DCs

D

CD25

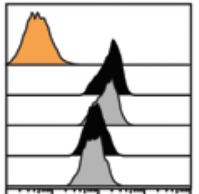

CD80
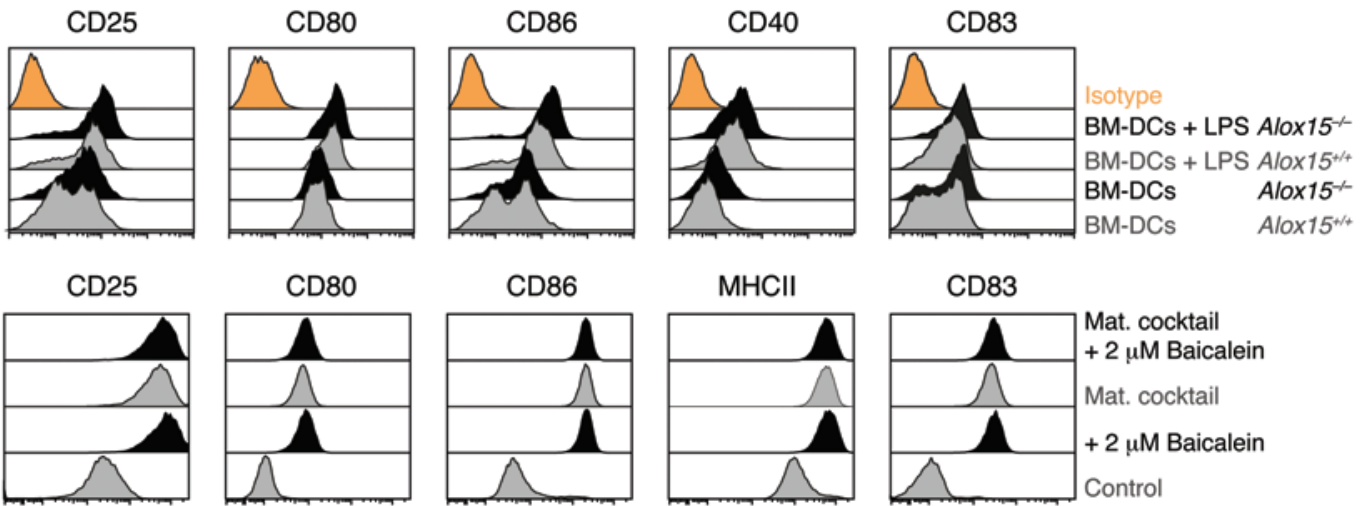

Figure 2. 12/15-LO regulates the maturation process of DCs. Flow cytometry-based analysis of (A) the expression of MHC class II molecules (MHCII) or (B)

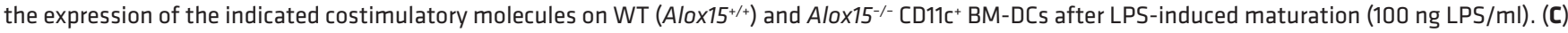
Comparison of surface marker expression levels on CD11 ${ }^{+}$BM-DCs by mean fluorescence intensity. (D) Effect of the 15-LO inhibitor Baicalein on the expression of the indicated surface molecules on human moDCs in the absence and presence of maturation (mat) cocktail. Data are representative of at least 3 independent experiments $(n=3)$. Error bars represent SEM. ${ }^{*} P<0.05,{ }^{* *} P<0.005$, Student's $t$ test.

glutamate-cysteine ligase $(\mathrm{Gclc})$. ChIP experiments showed that DNA binding of NRF2 to the promoters of these genes was greatly

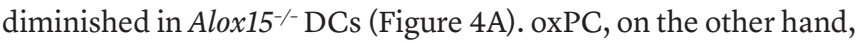
strongly induced association of NRF2 to the promoters of these genes and triggered a robust increase in their mRNA expression in WT DCs but not $\mathrm{Nrf2}^{2--}$ DCs (Figure 4, B and C). To determine whether NRF2 was involved in the oxPC-induced block of DCs, we analyzed the effects of oxPC on the expression of costimulatory molecules in WT and Nrf2- DCs. Indeed, absence of NRF2 abrogated the inhibitory effects of oxPC (Figure 4D), suggesting that NRF2 functions as a key mediator of the oxPC-mediated immune-modulatory effects in DCs. 

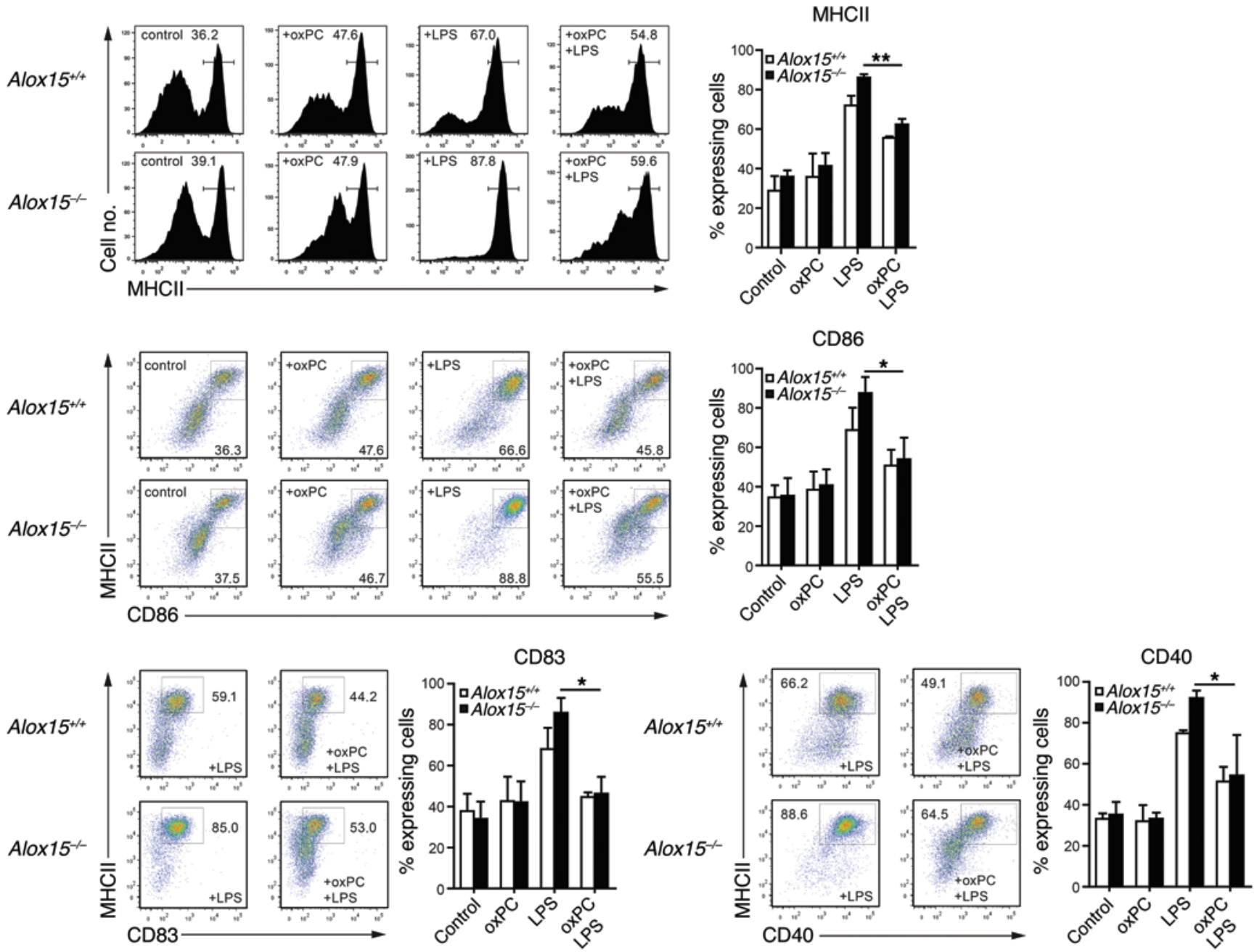

Figure 3. oxPC reverses the increased activation of 12/15-LO-deficient DCs. Flow cytometry-based analysis of the expression of MHC class II molecules and the indicated costimulatory molecules or activation markers on WT and Alox15 ${ }^{-1-}$ CD11 ${ }^{+}$BM-DCs after LPS-induced maturation (100 ng LPS/ $\mathrm{ml})$ in the absence and presence of oxPC $(50 \mu \mathrm{g} / \mathrm{ml})$. Data shown are representative of at least 3 independent experiments $(n=3)$. Error bars represent SEM. ${ }^{*} P<0.05,{ }^{*} P<0.005$, Student's $t$ test.

DC-intrinsic 12/15-LO activity attenuates Th17 $T$ cell differentiation. During their maturation process, DCs start to express several cytokines, which subsequently shape the developing $\mathrm{T}$ cell response. Among other DC-derived cytokines, DCderived IL-12 family members, such as IL-12 and IL-23, contribute to the development and maintenance of Th1 and Th17 T cells, respectively (16). The analysis of the cytokine expression profile of Alox $15^{-/}$DCs showed multiple alterations in comparison to that for WT DCs. Notably, Alox $15^{-}$DCs displayed a shift in the mRNA and protein expression of different IL-12/IL-23 subunits and increased expression of the IL-23-specific subunit p19, whereas expression of the subunit $\mathrm{p} 40$ (shared by IL-12 and IL-23) was unaltered and the IL-12-specific subunit p35 was decreased (Figure 5, A and B).

To determine the functional consequences of DC-intrinsic 12/15-LO activity on the $\mathrm{T}$ cell response, we analyzed allogeneic mixed-leukocyte reactions with DCs isolated from WT and Alox $15^{-/}$mice (C57BL/6 background) and lymphocytes isolated from BALB/c mice. Despite the increased maturation of Alox $15^{-1-}$ DCs, we did not observe major differences in their T cell stimu- latory capacity (Supplemental Figure 3). Since Alox15-/ DCs displayed an increased expression of IL-23p19, we subsequently determined the effect of 12/15-LO expression on the differentiation of Th17 T cells. Therefore, we cocultivated DCs together with $\mathrm{CD}_{4}{ }^{+} \mathrm{T}$ cells that were exposed to Th1-, Th2-, Th17-, or regulatory $\mathrm{T}$ cell-skewing conditions. Absence of $12 / 15-\mathrm{LO}$ in DCs hardly affected Th1 or Th2 differentiation but resulted in an increased differentiation of Th17 cells, whereas regulatory $\mathrm{T}$ cell development was diminished (Figure 5C). Addition of oxPC, in turn, interfered with the differentiation of Th17 cells restoring a regular Th17 frequency (Figure 5D). Interestingly, oxPC also exerted an inhibitory influence on Th17 differentiation when DCs were absent (Figure 5E), which suggests that lipid oxidation products of 12/15-LO such as oxPC can act as direct inhibitors of Th17 differentiation.

Alox $15^{-1-}$ mice display an increased differentiation of Th17 cells and an aggravation of $T$ cell-dependent autoimmune responses. To determine the consequences of the altered maturation process of Alox $15^{-/}$DCs in vivo, we decided to study the murine EAE model in WT and Alox15-- mice. EAE is considered as a prototypic Th17- 
A $\quad$ A Alox $15^{+/ 4} \quad$ Alox $15^{-1}$
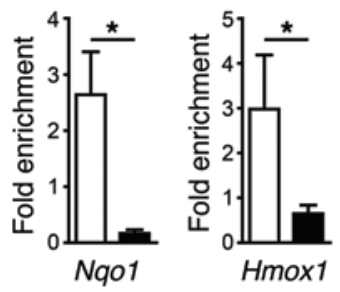
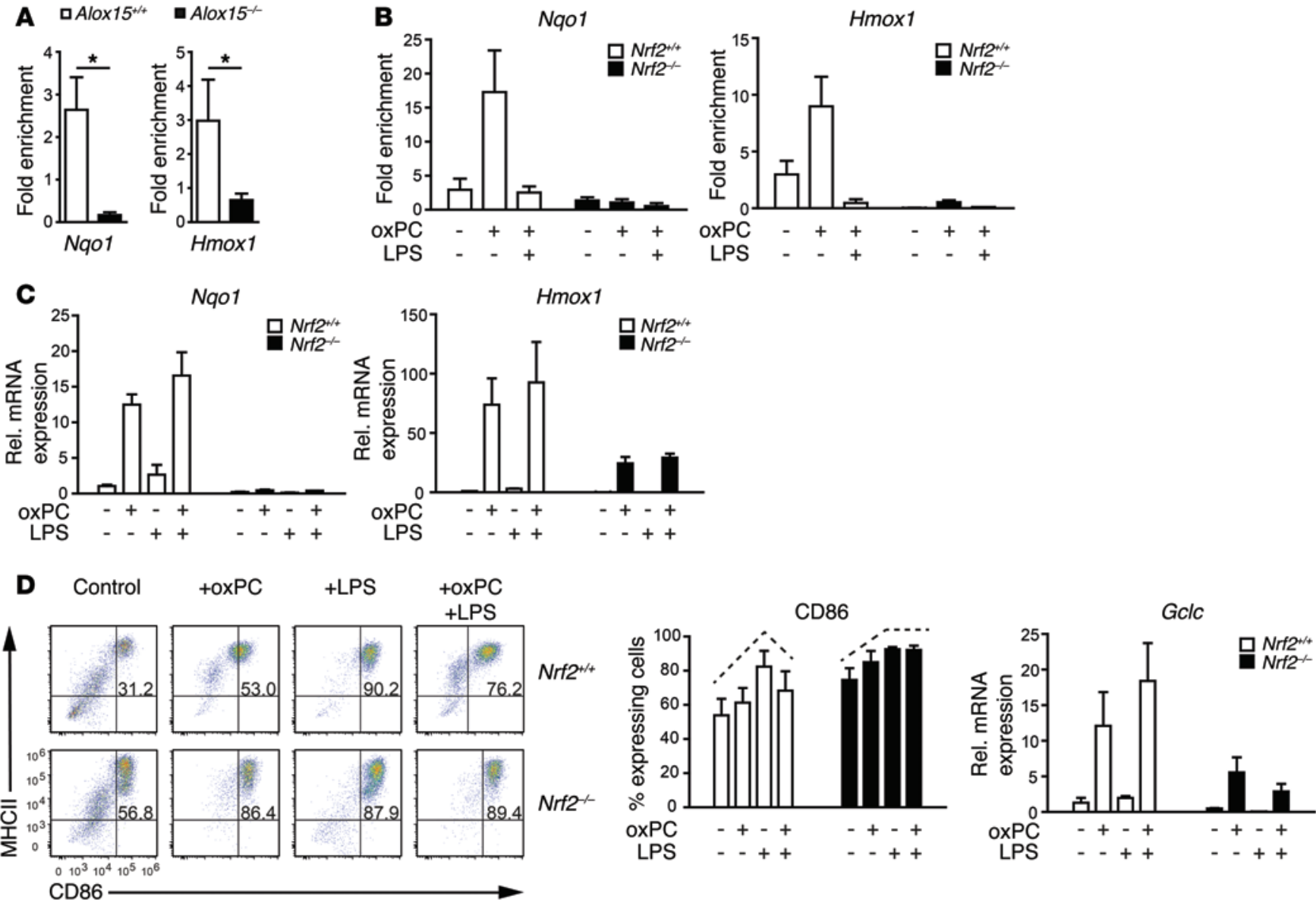

CD83
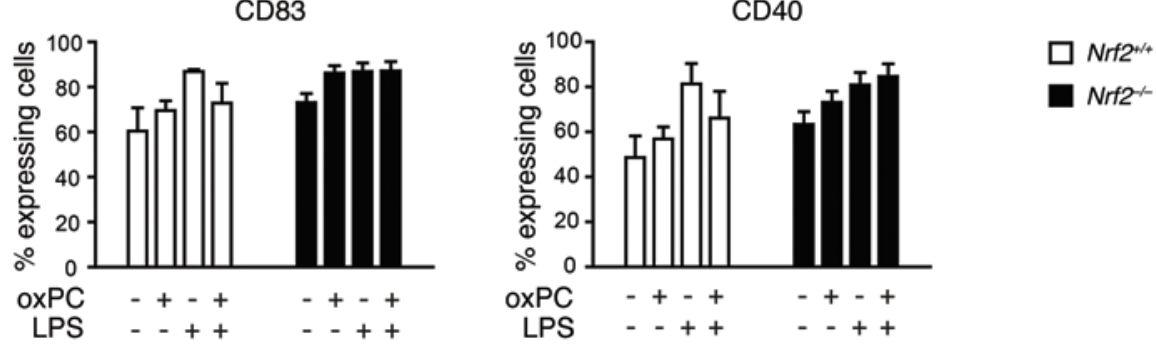

Figure 4. NRF2 mediates the immune-modulatory effects of 12/15-LO-derived oxidized phospholipids. (A) ChIP-based analysis of the binding of NRF2 to the Nqo1 and Hmox1 promoter in WT and Alox15 ${ }^{-1-}$ DCs. (B) ChIP-based analysis of the oxPC-induced binding of NRF2 to the Nqo1 and Hmox1 promoter in WT and Nrf2 $2^{-/-}$DCs. (C) oxPC-induced expression of Nqo1, Hmox1, and Gclc mRNA expression in WT and Nrf2 $2^{-/-}$DCs. (D) Flow cytometry-based quantification of the oxPC-induced inhibition of the expression of the indicated surface molecules on WT and Nrf2 $2^{-1-}$ DCs. Data shown are representative of at least 3 independent experiments $(n=3)$. Error bars represent SEM. ${ }^{*} P<0.05$, Student's $t$ test.

mediated autoimmune disease (17). After immunization with MOG peptide, newly primed autoreactive T cells invade the CNS, resulting in CNS inflammation and progressive paralysis.

Analysis of spleens and lymph nodes showed expression of $12 / 15-\mathrm{LO}$ in different DC subsets, whereas neither T nor B cells expressed this enzyme (Figure 6A and Supplemental Figure 4, $\mathrm{A}-\mathrm{C}$ ). Following immunization, DC maturation was paralleled by a gradual downregulation of 12/15-LO expression (Figure 6B). In accordance with our in vitro data, DCs in Alox $15^{-/-}$animals displayed an enhanced maturation status, as reflected by increased expression of MHC class II molecules (Figure 6C). This phenotype was more pronounced in the moDC and myeloid $\mathrm{DC}\left(\mathrm{CD} 11 \mathrm{c}^{+} \mathrm{CD} 11 \mathrm{~b}^{+} \mathrm{CD} 8^{-}\right)$subsets, whereas the maturation status of lymphoid $\left(\mathrm{CD} 11 \mathrm{c}^{+} \mathrm{CD} 11 \mathrm{~b}^{-} \mathrm{CD} 8^{+}\right)$DCs showed no major changes. Furthermore, Alox $15^{-/}$mice displayed an altered cytokine expression profile after induction of disease. Analysis of the mRNA expression profile in the draining lymph nodes of these Alox $15^{-/-}$mice revealed a significant increase in the expression of the IL-23 subunit p19, whereas expression levels of the IL-12/IL-23 subunits $p 35$ and $p 40$ were not significantly changed (Figure 6D). In accordance with our data on 12/15-LO protein expression in the different DC subsets, expression of Alox15 mRNA decreased after disease initiation. Moreover, we observed increased levels of $I l 1 b$ and an elevated expression of the Th17-specific transcription factor Rorgt in lymph nodes of Alox $15^{-/-}$mice. 
A

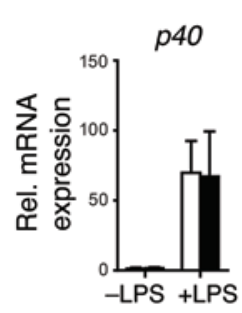

ㅁlox $15^{+/ *}$

- Alox $15^{-1-}$ p35

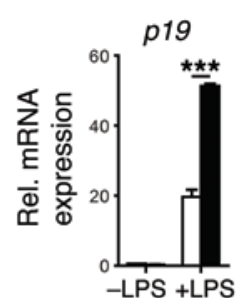

B

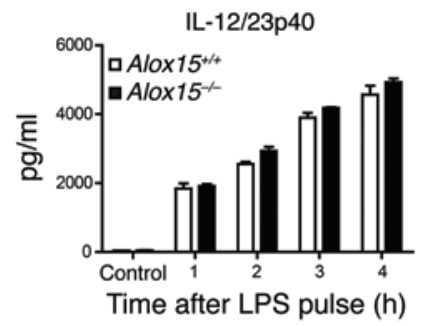

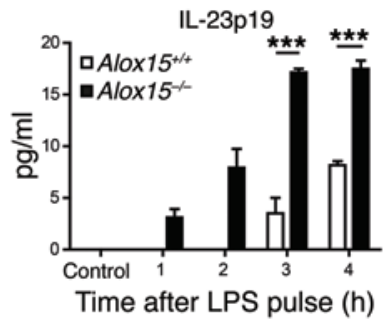

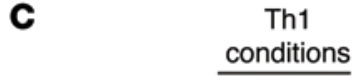

$+\mathrm{BMDC}^{++}$

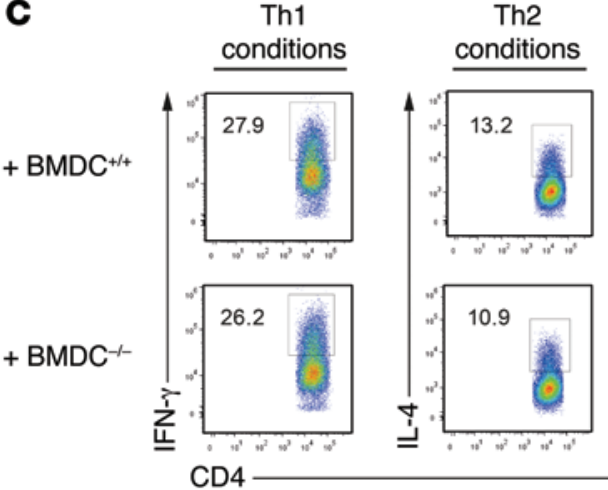

D

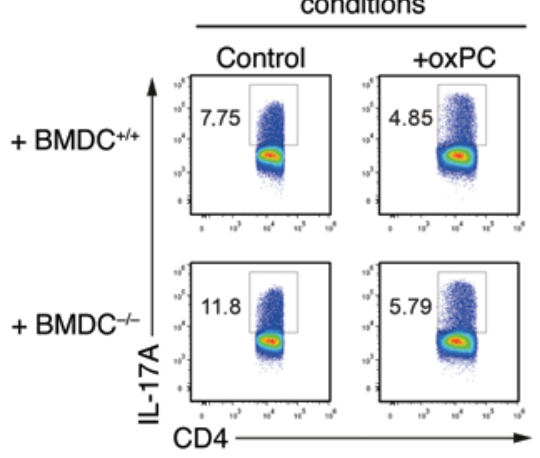

Th17

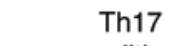

conditions

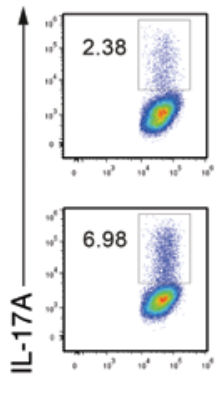

Treg conditions

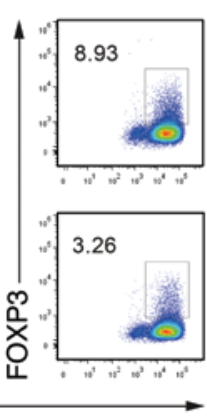

\section{E}

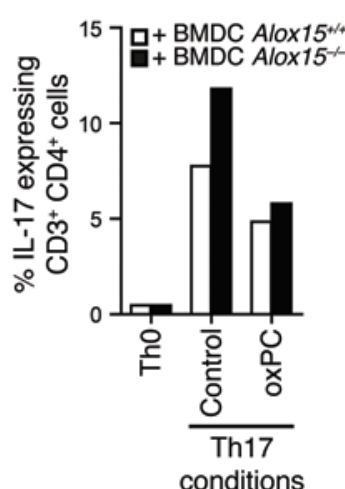

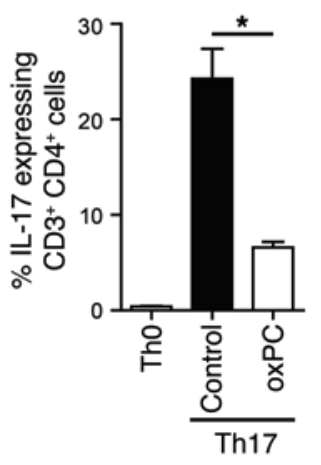

conditions

Figure 5. DC-derived 12/15-LO regulates Th17 $\mathrm{T}$ cell differentiation. (A) Real-time PCR-based quantification of mRNA expression levels of the indicated

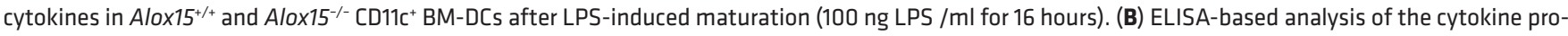
duction by BM-DCs from Alox $15^{+/+}$and Alox $15^{-/-}$mice. Cells were pulse stimulated with LPS $(100 \mathrm{ng} / \mathrm{ml})$ for 6 hours, and the subsequent cytokine secretion was determined at the indicated time points. (C) Flow cytometric analysis of the frequency of the indicated Th cell subsets after cocultivation of CD4+ $\mathrm{T}$ cells

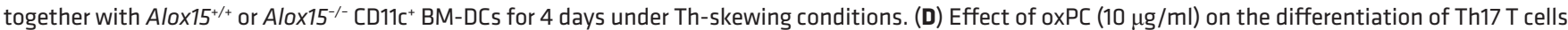
during cocultivation of CD4+ T cells together with Alox15 $5^{+/+}$or Alox15 ${ }^{-/-}$CD11 $\mathrm{c}^{+} \mathrm{BM}$-DCs under Th17-skewing conditions. (E) Effect of oxPC (10 $\left.\mu \mathrm{g} / \mathrm{ml}\right)$ on the differentiation of Th17 T cells under Th0- or Th17-skewing conditions in the absence of BM-DCs. Data shown are representative of at least 3 independent experiments $(n=3)$. Error bars represent SEM. ${ }^{*} P<0.05,{ }^{* * *} P<0.0005$, Student's $t$ test.

We subsequently analyzed the distribution of different $\mathrm{T}$ cell subsets in the spleens of WT and Alox15\% mice, which were harvested 10 days after the initial immunization. Flow cytometry showed a significant increase in the number of Th17 T cells in Alox $15^{-/}$mice, whereas the distribution of other $\mathrm{T}$ cell subsets, such as $\mathrm{FOXP}^{+}$regulatory $\mathrm{T}$ cells, Th1 cells, or Th2 $\mathrm{T}$ cells, was not changed (Figure 6E). In accordance with these findings, the clinical

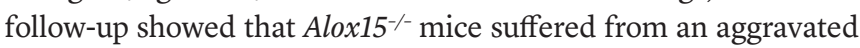
form of EAE. In a "classical" EAE model, which included application of pertussis toxin, we observed an increased clinical score in these mice (Figure 6F). In a modified EAE protocol, performed without the administration of pertussis toxin, both the clinical score and the disease incidence were significantly increased in Alox15 ${ }^{-/}$mice compared with those in WT controls (Figure 6G).

\section{Discussion}

In the current manuscript, we describe a so far unrecognized role of the lipid-oxidizing enzyme 12/15-LO during the regulation of DC maturation and the modulation of the adaptive immune response. Deletion of the gene encoding 12/15-LO in murine BM-DCs resulted in enhanced DC maturation upon TLR ligation, while inhibition of the human ortholog 15-LO triggered a 
A
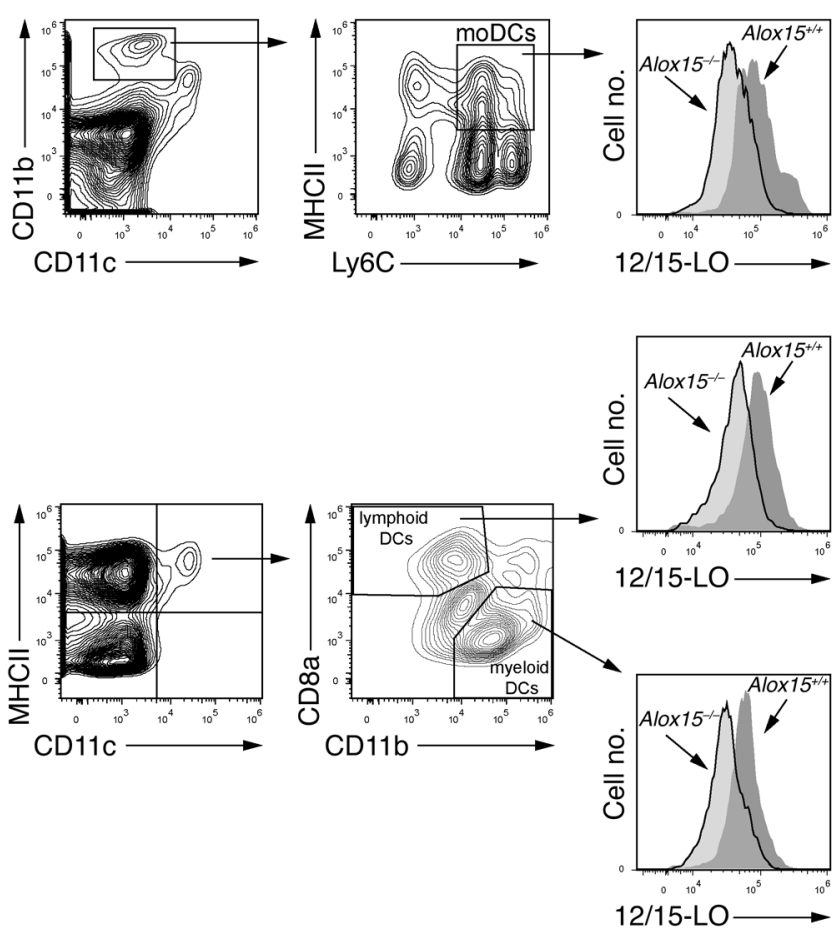

B EAE immun., spleen

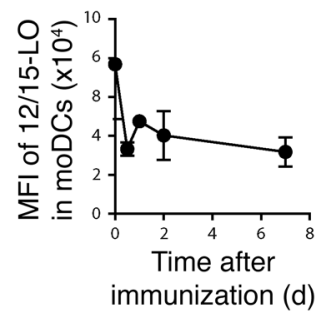

C EAE immun., LNs
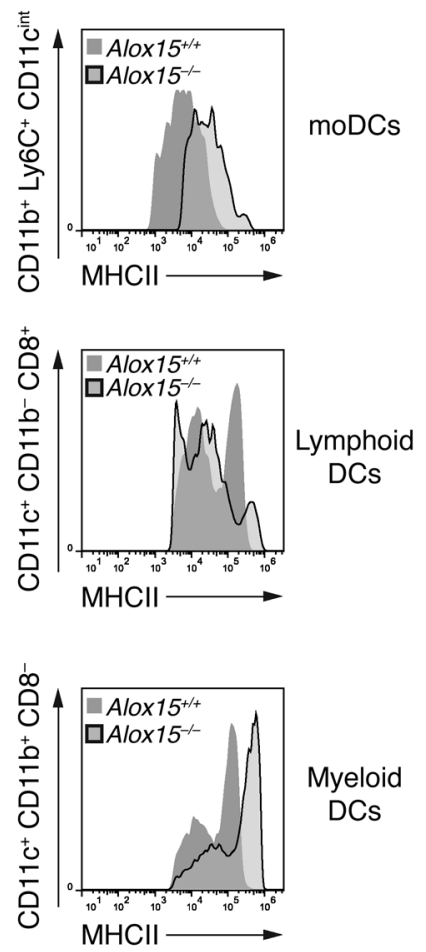

D
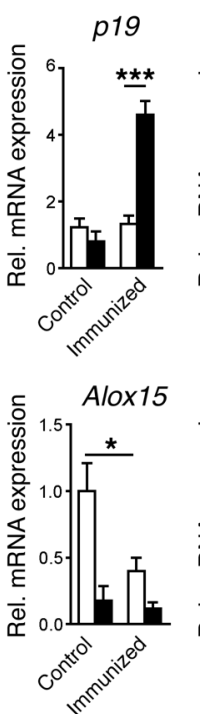

$\square$ Alox 15 $\quad$ Alox $15^{-1 /}$
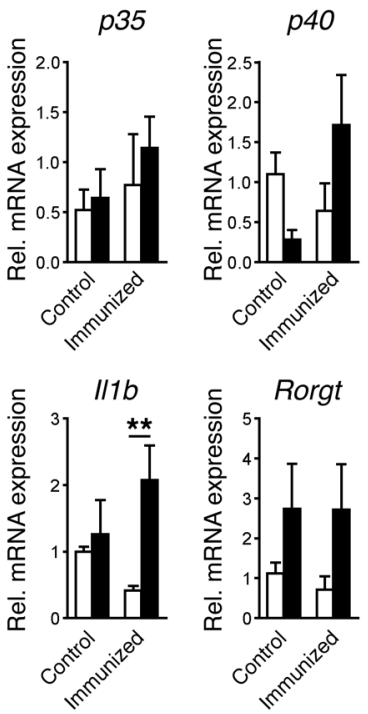

E

IL-17

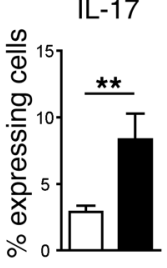

FOXP3

Alox $15^{+/ t}$

- Alox $15^{-1}$

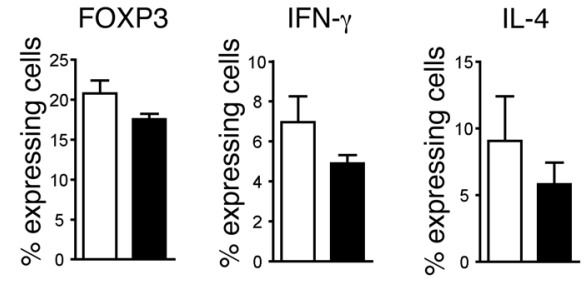

IL-4
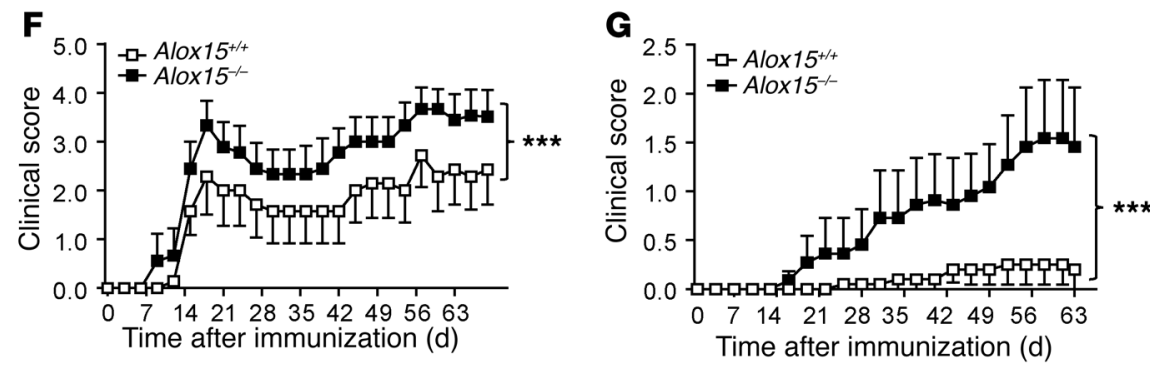

Figure 6. Deletion of 12/15-LO results in increased DC maturation and Th17 T cell differentiation as well as an aggravated form of EAE. (A) Flow cytometry-based measurement of the 12/15-LO expression in indicated splenic DC subsets during steady state and (B) follow-up of 12/15-LO expression in moDCs after induction of EAE. (C) Flow cytometric analysis of MHC class II molecule expression on moDC, lymphoid DC, and myeloid DCs, and (D) quantification of the mRNA expression profile of indicated cytokines, including Alox15 and Rorgt, in draining lymph nodes of WT and Alox15 ${ }^{-/-}$mice 3 days after induction of immunized (EAE) mice compared with nonimmunized mice (control). (E) Flow cytometric analysis of the frequency of IL-17-expressing Th17 T cells, FOXP3expressing regulatory T cells, IFN- $\gamma$-expressing Th1 T cells, and IL-4-expressing Th2 T cells in the spleens of WT or Alox15 $5^{-1-}$ mice 10 days after induction of EAE. Data shown are representative of at least 3 independent experiments. Error bars represent SEM. ${ }^{*} P<0.05,{ }^{* *} P<0.005$, ${ }^{* * *} P<0.0005$, Student's $t$ test. (F and $\mathbf{G})$ Analysis of the EAE severity score in WT and Alox $15^{-1-}$ mice after immunization with MOG peptide in the $(\mathbf{F})$ presence or $(\mathbf{G})$ absence of pertussis toxin. Mean clinical scores were determined based on at least 7 mice per group $(n=7)$. ${ }^{* * *} P<0.001$, Mann-Whitney's $U$ test.

spontaneous maturation of moDCs. This difference in effect size might be due to intrinsic cellular differences between human blood monocyte-derived DCs and murine BM-DCs or to the slight shift in the catalytic preferences and product profile of murine
12/15-LO and human 15-LO (18). 12/15-LO-derived phospholipid oxidation products were, in turn, able to block LPS-induced DC maturation. Together, these data suggest an endogenous regulatory mechanism that involves the generation of $12 / 15-\mathrm{LO}$-derived 
phospholipid oxidation products that provide a critical activation threshold to limit the spontaneous and excessive maturation of DCs. The observed downregulation of 12/15-LO during regular LPS-induced maturation might instead be a prerequisite for a controlled maturation of DCs.

A regulatory effect of oxidized phospholipids on human moDCs has been previously described $(19,20)$, although a potential source of these lipids as well as the downstream targets have remained largely elusive in these studies. Our current data suggest that $12 / 15-\mathrm{LO}$ acts as an important enzymatic source for such bioactive oxidized phospholipids in DCs that, in turn, control the DC's maturation state via the transcription factor NRF2.

Previous analyses of the immune response in hyperlipidemic Apoe ${ }^{-/}$mice, which show elevated levels of oxidized LDLs in their sera, have revealed an inhibitory effect of oxidized LDLs on the TLR-induced maturation of CD8 ${ }^{-}$DCs in vivo as well as on the development of Th17-mediated autoimmune disease $(13,21,22)$. Notably, 12/15-LO is the major enzyme involved in the oxidation of LDL particles in such hyperlipidemic mice (23) and the biologic activity of oxidized LDL is strongly linked to LDL-associated oxidized phospholipids (24). Hence, these findings are well in accordance with our current data and support the concept of a regulative role of lipid oxidation during DC maturation and initiation of the adaptive immune response. Our present study shows that the inhibitory effect of $12 / 15-\mathrm{LO}$-mediated lipid oxidation on the maturation of the CD8- DC subsets and the Th17 response is independent from hyperlipidemia, as the mice in our experiments were not fed a highfat diet. Therefore, both LDL-derived and endogenous cell membrane-derived lipids can act as the source for the 12/15-LO-mediated generation of lipid mediators that attenuate DC maturation.

Mechanistically, our data suggest that 12/15-LO-derived phospholipid oxidation products activate the transcription factor NRF2 and, thereby, feedback on the antioxidative response of the DC. Indeed, NRF2 not only acts as master regulator of the antioxidative response but was likewise shown to negatively control DC maturation (25-28).

Moreover, our current data show a regulatory role of 12/15LO-mediated enzymatic lipid oxidation on the regulation of the IL-12/IL-23 axis and the differentiation of Th17 T cells. While $12 / 15$-LO activity attenuated expression of the IL-12 family member IL-23, a cytokine that has been implicated in the maintenance of functional Th17 cells, 12/15-LO-derived lipid mediators also directly interfered with Th17 differentiation. 12/15-LO was previously shown to regulate IL-12 expression in macrophages, in which deletion of this enzyme results in a reduced production of this cytokine $(29,30)$. Our current data confirms a 12/15-LO-mediated regulation of IL-12p35 in DCs and additionally demonstrates a reciprocal regulation of the IL-12/IL-23 balance by $12 / 15-\mathrm{LO}$ in such professional antigen-presenting cells, suggesting a slightly differential role of this enzyme in DC and macrophage biology.

In addition, COX-2-derived prostaglandin $\mathrm{E}_{2}\left(\mathrm{PGE}_{2}\right)$ was shown to regulate the IL-12/IL-23 axis in DCs (31). In contrast to 12/15-LO, however, this COX-2 metabolite induced IL-23p19 and inhibited IL-12p35, thus indicating an inverse regulation of the IL-12/IL-23 ratio by COX-2 and 12/15-LO. The fact that COX-2 and $12 / 15-\mathrm{LO}$ both metabolize polyunsaturated fatty acids enables a potential substrate competition between the two enzymes.
Although a substrate shift could thus theoretically contribute to the increase in IL-23/IL-12 ratio in the absence of $12 / 15-\mathrm{LO}$, we did not detect an increase in $\mathrm{PGE}_{2}$ levels in Alox $15^{-/-}$DCs (Supplemental Figure 5, A and B). In addition, indomethacin-induced inhibition of COX-2 activity did not ameliorate the increase in Il23p19 expression or the decrease in Il12p35 expression in Alox $15^{-/-}$DCs (Supplemental Figure 5, C and D), arguing against a major role of substrate competition during the 12/15-LO-mediated regulation of the IL-12/IL-23 axis.

The increased differentiation of Th17 T cells in Alox15 $1 /$ mice cumulated in an exacerbated form of EAE as a prototypical Th17driven autoimmune disease. These data support previous findings that described an enhanced susceptibility of Alox $15^{-/-}$mice to the development of this disease (32), although the authors of this previous study did not address the underlying mechanism. In accordance, NRF2-deficient mice develop an exacerbated form of EAE as well, whereas pharmacological compounds activating this transcription factor attenuate this disease in mice and were already successfully tested in the treatment of multiple sclerosis in humans (33-36).

Interestingly, 12/15-LO has also been implicated in the development of type 1 diabetes and atherosclerosis, in which deletion of Alox15 results in reduced disease incidence (37). Although the exact role of $12 / 15-\mathrm{LO}$ in the pathogenesis of type 1 diabetes remains largely elusive, 12/15-LO-induced oxidative stress and the direct cytotoxic effect of 12-HETE seem to contribute to mitochondrial dysfunction and altered insulin secretion. As type 1 diabetes represents a prototypical Th1-driven disease, the observed reduction of the IL-12/IL-23 ratio might additionally contribute to attenuation of this disease in Alox $15^{-/-}$mice.

Taken together, our current study provides evidence for a central role of enzymatic lipid oxidation during the modulation of DC function and the shaping of an adaptive immune response. These insights might result in the identification of novel targets for the treatment of autoimmune diseases, such as multiple sclerosis and rheumatoid arthritis.

\section{Methods}

Animals. Animal experiments were approved by the government of Mittelfranken. Mice were housed in the animal facility of the University of Erlangen-Nuremberg. Alox $15^{-/}$mice were purchased from The Jackson Laboratory; C57BL/6 mice were purchased from Charles River Laboratories. All experiments comparing Alox $15^{-/-}$and WT mice and DCs were confirmed using littermates. NRF2-deficient ( $\left.\mathrm{Nrf}^{-/-}\right)$ mice were previously described (38).

Generation of murine BM-DCs. Bone marrow cells were flushed from the femurs and tibias of $\mathrm{C} 57 \mathrm{BL} / 6$ or $\mathrm{Alox} 15^{-/-}$mice and cultured for 8 days in R10 medium at a concentration of $2 \times 10^{6}$ cells per $10-\mathrm{cm}$ dish (Falcon, no. 1029, bacterial quality) and granulocyte macrophage colony-stimulating factor (GM-CSF). R10 culture medium is composed of RPMI 1640 (Lonza) supplemented with penicillin (100 $\mathrm{U} / \mathrm{ml}$, Lonza), streptomycin (100 $\mathrm{mg} / \mathrm{ml}$, Lonza), L-glutamine (2 $\mathrm{mM}$, Lonza), 2-mercaptoethanol (50 mM, Sigma-Aldrich), and 10\% heat-inactivated FCS (PAA Laboratories). GM-CSF supernatant (1:10) from a cell line transfected with the murine GM-CSF gene was used (39). At day 3, a volume of $9 \mathrm{ml} \mathrm{R10} \mathrm{medium} \mathrm{containing} 1 \mathrm{ml} \mathrm{GM-CSF}$ supernatant was added to the cultures. $50 \%$ of the culture supernatant 
was removed at day 6, and cells were fed again with fresh $10 \mathrm{ml}$ of 1:10 diluted R10 medium containing GM-CSF supernatant. On day 8, nonadherent cells, which were $>95 \% \mathrm{CD} 11 \mathrm{c}^{+}$, were harvested and used for the different experiments (40).

Maturation of murine DCs and treatments. On day 8, maturation of BM-DC cultures was induced by overnight treatment with $100 \mathrm{ng} / \mathrm{ml}$ LPS. In the case of LPS pulse stimulation, BM-DCs were incubated with $100 \mathrm{ng} / \mathrm{ml}$ LPS for 6 hours. Following incubation, medium was removed and adherent cells were washed twice with PBS. Cells were supplied with fresh R10 medium an incubated at $37^{\circ} \mathrm{C}$ until supernatants were harvested for determination of the cytokine content.

For lipid pretreatment, BM-DCs were incubated for 4 hours at $37^{\circ} \mathrm{C}$ before addition of maturation stimuli. oxPC was used at $50 \mu \mathrm{g} / \mathrm{ml}$. For COX-2 inhibition, BM-DCs were pretreated with $10 \mu \mathrm{M}$ indomethacin (4 hours, $37^{\circ} \mathrm{C}$ ) prior to LPS-induced maturation.

Generation of human moDCs. Human moDCs were generated as described previously (41). Briefly, peripheral blood mononuclear cells (PBMCs) were isolated from leukoreduction system chambers of healthy donors by density centrifugation using Lymphoprep (AxisShield PoC). PBMCs were seeded on tissue culture dishes (BD Falcon) for 1 hour. The nonadherent fraction was washed off after 1 hour with RPMI 1640 without any supplement. The adherent cell fraction was cultured for 4 days in DC medium consisting of RPMI 1640 (Lonza) supplemented with $1 \%$ (vol/vol) of each of the following: heat-inactivated human serum type AB (Lonza), Penicillin/Streptomycin/L-Glutamine (PAA Laboratories), and $10 \mathrm{mM}$ HEPES (Lonza) as well as 800 $\mathrm{IU} / \mathrm{ml}$ (day 0) or $400 \mathrm{IU} / \mathrm{ml}$ (day 3) recombinant human GM-CSF and $250 \mathrm{IU} / \mathrm{ml}$ (day 0 and 3) recombinant IL-4 (both Cell Genix). On day 4, immature DCs were used for further experiments.

Maturation of human DCs and treatment with Baicalein. Maturation of DCs was induced by the addition of a maturation cocktail consisting of $200 \mathrm{U} / \mathrm{ml} \mathrm{IL}-1 \beta$ (Cell Genix), 1,000 U/ml IL-6 (Cell Genix), $10 \mathrm{ng} /$ $\mathrm{ml} \mathrm{TNF-} \alpha$ (Beromun, Boehringer Ingelheim), and $1 \mu \mathrm{g} / \mathrm{ml} \mathrm{PGE}_{2}$ (Prostin $E_{2}$, Pfizer) for 24 hours. Immature DCs were either incubated with $2 \mu \mathrm{M}$ Baicalein (Sigma-Aldrich) formulated in DMSO or treated with 2 $\mu \mathrm{M}$ Baicalein and subsequently matured as indicated above. DMSO on its own was used as control.

Phospholipid preparation. Generation of oxPC has been previously described $(42,43)$. oxPC was generated by air oxidation of the corresponding nonoxidized precursor phosphatidylcholine (PAPC) until $20 \%$ of PAPC remained intact, while the rest transformed into oxidized products. The extent of oxidation was monitored by thin-layer chromatography, and the analysis of the oxidation products was performed by flow-injection electrospray ionization mass spectrometry. Phospholipid concentration was determined by phosphorus assay. Phospholipids were dissolved in chloroform and stored at $-80^{\circ} \mathrm{C}$ until use. Immediately before an experiment, chloroform solutions of oxPC were evaporated under a stream of argon and resuspended in culture medium by vigorous vortexing for 30 seconds.

Cell preparation and flow cytometry. To compare 12/15-LO expression in moDCs and conventional DCs, inguinal lymph nodes and spleens were harvested. Further, lymph nodes and spleens were digested for 25 to 30 minutes at $37^{\circ} \mathrm{C}$ with collagenase-DNase and then treated for 5 minutes with EDTA to disrupt T cell-DC complexes. After fixation with $4 \% \mathrm{PFA}$ and surface staining, cells were permeabilized using Fixation/Permeabilization solution (eBioscience) and blocked with Permeabilization buffer (Perm; eBioscience) contain- ing $10 \%$ goat serum. A polyclonal rabbit anti-mouse antibody against 12/15-LO (Abcam) was used in Perm/5\% goat serum for 60 minutes at $4^{\circ} \mathrm{C}$; for isotype control, $5 \%$ rabbit serum was used instead of primary antibody. After intense washing with Perm, secondary antibody against rabbit IgG (Dianova) was added at 1:400 for 30 minutes at $4^{\circ} \mathrm{C}$.

For analysis of surface markers, cells were stained in PBS containing $5 \% \mathrm{FCS}$ for 30 minutes at $4^{\circ} \mathrm{C}$. Cells were stained in various combinations of mAbs. For human moDCs, the following surface molecules were used: CD11c (B-ly6), CD25 (M-A251), CD86 (2331), and CD83 (HB15e) from BD Pharmingen; CD80 (2D10), CD14 (M5E2), and MHCII (L243) from BioLegend; and LIVE/DEAD (Life Technologies). For murine cells, we used the following: CD8a (53-6.7), CD11b (M1/70), CD25 (3C7), CD40 (3/23), CD86 (GL-1), Ly6C (HK1.4.), and MHCII (M5/114.15.2) from BioLegend; CD11c (N418), CD80 (16-10A1), and CD83 (Michel-19) from eBioscience for DCs; and CD3 (145-2C11) and CD4 (RM4-5) from eBioscience for T cells. For intracellular staining with $\alpha$ IL-17A (TC11), $\alpha$ FOXP3 (MF-14), $\alpha$ IFN- $\gamma$ (XMG1.2), or $\alpha \mathrm{IL}-4$ (11B11), all from BioLegend, T cells were stimulated for 5 hours with phorbol 12-myristate 13-acetate (PMA) and ionomycin in the presence of monensin before being stained according to the manufacturer's instructions (eBioscience). The exclusion of dead cells was performed by staining with Fixable Viability Dye (eBioscience) in parallel to surface staining. Flow cytometry data were acquired on a Gallios Flow Cytometer (Beckman Coulter) or FACScan (BD) and were analyzed with FlowJo software (Treestar).

ELISA and EIA. To determine supernatant concentrations of IL-23p19 and IL-12/23p40 (R\&D Systems), as well as PGE (Cayman), assays were performed according to the instructions in the manufacturer's protocols.

Western blot analysis. Cells and tissues were homogenized and lysed in Laemmli buffer. Protein concentration was determined using a RC/DC Protein Quantification Kit (Bio-Rad). Proteins were separated by electrophoresis in 10\% SDS polyacrylamide gels. Proteins were blotted onto polyvinylidene difluoride membranes and, after blocking with $5 \%$ dry milk/0.1\% Tween 20 , incubated with primary and secondary peroxidase-conjugated antibodies and consequent chemiluminescent detection.

Quantification of PAPC-OH and PAPC-OOH in BM-DCs. Nonadherent BM-DCs $\left(\sim 3 \times 10^{6}\right.$ cells $)$ from WT and KO animals were harvested on ice, centrifuged, and washed twice with PBS supplemented with $2 \mathrm{mM}$ EDTA and 0.01\% butylated hydroxytoluene (PBS/BHT/ EDTA). The suspensions were transferred into cryovials overlaid with argon and stored at $-80^{\circ} \mathrm{C}$. For cell culture supernatants, cells were centrifuged at $400 \mathrm{~g}$, and $500 \mu \mathrm{l}$ of the liquid phase was collected and supplemented with BHT to $0.01 \%$ and EDTA to $2 \mathrm{mM}$. Quantification of different 12/15-LO-derived oxidation products in WT and Alox15 BM-DCs was performed by liquid chromatography_tandem mass spectrometry as previously described (10).

Quantitative real-time PCR. Real-time PCR was performed as previously described (44). RNA was isolated using TRIzol reagent (Invitrogen). One microgram of total RNA was reverse transcribed with human leukemia virus reverse transcriptase using the Gene Amp RNA PCR Kit (Applied Biosystems) and oligo(dT) primers. mRNA levels were normalized to $\beta$-actin expression in the case of tissue or Gapdh in the case of BM-DC cultures. The following primer sequences were used: Gapdh: CTACACTGAGGACCAGGTTGTCT (sense), CAGGAAATGAGCTTGACAAAGTT (antisense); $\beta$-actin, 
TGTCCACCTTCCAGCAGATGT (sense), AGCTCAGTAACAGTCCGCCTAGA (antisense); Alox15: CTCTCAAGGCCTGTTCAGGA (sense), GTCCATTGTCCCCAGAACCT (antisense); Il23p19: GACCCACAAGGACTCAAGGA (sense), TAGAACTCAGGCTGGGCATC (antisense); Il12p35: ATGACCCTGTGCCTTGGTAG (sense), TCTCCCACAGGAGGTTTCTG (antisense); Il12/23p40: AGGTGCGTTCCTCGTAGAGA (sense), AAAGCCAACCAAGCAGAAGA (antisense); Il1b: CAGGCAGGCAGTATCACTCA (sense), AGGTGCTCATGTCCTCATCC (antisense); Rorgt: CCACTGCATTCCCAGTTTCT (sense), CGTAGAAGGTCCTCCAGTCG (antisense); Hmox1: GAATCGAGCAGAACCAGCCT (sense), AAGGAAGCCATCACCAGCTTA (antisense); Gclc: ATCTTCTGGCACAGCACGTT (sense), AACTGCACCTCCATTGGTCG (antisense); Nqo1: AGGCTGGTTtGagAgAgtgC (sense), CAgGATGCCACTCTGAATCGG (antisense); Cox2: TGAGTACCGCAAACGCTT (sense), CСАТTTCTTTCTCTCСTGTAA (antisense).

$T$ cell skewing and cocultures. Mouse splenic $\mathrm{CD} 4^{+} \mathrm{T}$ cells were isolated by immunomagnetic separation using EasySep (STEMCELL Technologies) and cultured in IMDM medium (Lonza). For cytokinemediated Th differentiation, $\mathrm{CD} 4^{+}$cells were cultured with plate-bound $5 \mu \mathrm{g} / \mathrm{ml} \alpha \mathrm{CD} 3$ antibody and IMDM medium supplemented with $3 \mu \mathrm{g} /$ $\mathrm{ml} \alpha \mathrm{CD} 28$ antibody (BioLegend). Cytokines and neutralizing antibodies were added at the following concentrations where indicated: (Th17) $5 \mathrm{ng} / \mathrm{ml}$ TGF- $\beta, 10 \mathrm{ng} / \mathrm{ml}$ IL-6 (BioLegend), $10 \mu \mathrm{g} / \mathrm{ml} \alpha \mathrm{IFN} \gamma$ antibody; (Th2) $20 \mathrm{ng} / \mathrm{ml} \mathrm{IL-2,} 50 \mathrm{ng} / \mathrm{ml} \mathrm{IL-4} \mathrm{(BioLegend);} \mathrm{(Th1)} 5 \mathrm{ng} / \mathrm{ml} \mathrm{IL-2,} 10$ $\mathrm{ng} / \mathrm{ml} \mathrm{IL}-12,10 \mu \mathrm{g} / \mathrm{ml} \alpha \mathrm{IL}-4$ (BioLegend); and (regulatory T cells) $5 \mathrm{ng} /$ $\mathrm{ml}$ IL-2, $5 \mathrm{ng} / \mathrm{ml}$ TGF- $\beta$ (BioLegend); for Th0 conditions, no cytokines or blocking antibodies were added. For assays of BM-DC-mediated T cell differentiation, DC were generated as described above, followed by LPS (100 ng/ml) maturation for 14 hours. DCs were washed extensively and cultured with enriched CD4 ${ }^{+} \mathrm{T}$ cells at a ratio of 1:5 in the appropriated T cell-skewing conditions supplemented with or without $10 \mu \mathrm{g} / \mathrm{ml}$ oxPC. After 4 days of culture, T cells were collected and stimulated with PMA and ionomycin (Sigma-Aldrich) plus monensin (BD Biosciences) for intracellular cytokine staining.

Induction of EAE. Female C57BL/6 mice and KO mice were immunized subcutaneously with $50 \mu \mathrm{g}$ MOG peptide 35-55 (Charité Berlin) in $50 \mu \mathrm{l} \mathrm{H}_{2} \mathrm{O}$ emulsified in $50 \mu \mathrm{l} \mathrm{CFA}$, which was enriched with $10 \mathrm{mg} / \mathrm{ml}$ Mycobacterium tuberculosis (H37Ra, Difco/PD PharMingen) at day 0 in order to induce EAE. In addition, 200 ng pertussis toxin (List/Quadratech) was administered intraperitoneally at day 0 and 2. EAE paralysis of mice was scored as follows: 0 , no disease; 1 , tail weakness; 2 , paraparesis; 3 , paraplegia; 4 , paraplegia with forelimb weakness; 5 , moribund or death. In a modified procedure, EAE induction was performed without the additional administration of pertussis toxin.

ChIP. ChIP analysis was performed using the ChIP-IT High-Sensitivity Kit (Active Motif). BM-DCs were treated with $100 \mathrm{ng} / \mathrm{ml}$ LPS or 50 $\mu \mathrm{g} / \mathrm{ml}$ oxPC in RPMI containing 10\% FCS. After 2 hours of incubation, cells were fixated and prepared for chromatin sonication according to the manufacturer's specifications. Chromatin shearing was performed using an EpiShear Probe Sonicator (Active Motif). Sonicated chromatin was incubated with antibodies ( $4 \mu \mathrm{g}$ per $30 \mu \mathrm{g}$ chromatin) against NRF2 (Cell Signaling, 12721) and IgG (Santa Cruz, sc-2027). Before immunoprecipitation, $5 \%$ of the extract volume was removed and served as an input. The antibody-bound protein/DNA complexes were immunoprecipitated through the use of protein $\mathrm{G}$ agarose beads and washed via gravity filtration. Following immunoprecipitation, the DNA crosslinks were reversed and the DNA was purified according to the ChIP protocol. Purified immunoprecipitated and input DNA were analyzed by PCR. The following primers were used: for Hmox1 promoter region containing putative ARE (-352/-338), TTAGGAATCCGGAGCTGTGC (sense), AGGGTTCAGTCTGGAGCAAC (antisense), and for NQO1 ARE (9510/9524), TCTAAGAGCAGAACGCAGCA (sense), AGTCACCTTTGCACGCTAGG (antisense). Data were expressed as fold enrichment of the ChIP samples relative to the IgG samples.

Study approval. For the generation of PBMCs and moDCs from leukoreduction system chambers of healthy donors, the positive vote from the local ethics committee of Mittelfranken was obtained (Re. no.: 4556).

Statistics. Data are shown as mean \pm SEM. Group mean values were compared by 2-tailed Student's $t$ test. Mean clinical scores of the EAE experiments were determined based on at least 7 mice per group, and statistical differences between the groups were compared by Mann-Whitney's $U$ test. The data shown are representative of at least 3 experiments generating similar results.

\section{Acknowledgments}

We thank Cornelia Stoll, Alexandra Klej, Lena Sandrock, and Ionela-Mariana Nagelreiter for excellent technical assistance. This study was supported by the Else-Kröner Fresenius Stiftung (to G. Krönke), the Deutsche Forschungsgemeinschaft (SCHE1583 to G. Schett; KR3523 to G. Krönke; FG 661 to G. Schett; SFB 643 to G. Schett, E. Zinser, and A. Steinkasserer; and SPP1468-IMMUNOBONE to G. Krönke and G. Schett), the ELAN-Program of the University of Erlangen-Nuremberg (to G. Krönke), the MASTERSWITCH project of the European Union (to G. Schett), the Interdisciplinary Centre for Clinical Research, Erlangen (to G. Krönke), the Fonds zur Förderung wissenschaftlicher Forschung (P22267-B11 to O.V. Oskolkova), and the NIH (R01 DK096076 to N. Leitinger).

Address correspondence to: Gerhard Krönke, University of Erlangen, Glueckstrasse 4a, Erlangen, UNK 91054, Germany. Phone: 49.0.9131.85.43012; E-mail: gerhard.kroenke@uk-erlangen.de.
1. Merad M, Sathe P, Helft J, Miller J, Mortha A. The dendritic cell lineage:ontogeny and function of dendritic cells and their subsets in the steady state and the inflamed setting. Annu Rev Immunol. 2013;31:563-604.

2. Ganguly D, Haak S, Sisirak V, Reizis B. The role of dendritic cells in autoimmunity. Nat Rev Immunol. 2013;13(8):566-577.

3. Ivanov I, et al. Molecular enzymology of lipoxygenases. Arch Biochem Biophys. 2010;503(2):161-174.
4. Kühn H, Römisch I, Belkner J. The role of lipoxygenase-isoforms in atherogenesis. Mol Nutr Food Res. 2005;49(11):1014-1029.

5. Cyrus T, et al. Disruption of the 12/15-lipoxygenase gene diminishes atherosclerosis in apo E-deficient mice. J Clin Invest. 1999;103(11):1597-1604.

6. Kühn H, O'Donnell VB. Inflammation and immune regulation by 12/15-lipoxygenases. Prog Lipid Res. 2006;45(4):334-356.
7. Serhan CN, Chiang N, Van Dyke TE. Resolving inflammation:dual anti-inflammatory and pro-resolution lipid mediators. Nat Rev Immunol. 2008;8(5):349-361.

8. Serhan CN, et al. Reduced inflammation and tissue damage in transgenic rabbits overexpressing 15-lipoxygenase and endogenous anti-inflammatory lipid mediators. J Immunol. 2003;171(12):6856-6865.

9. Krönke G, et al. 12/15-lipoxygenase counteracts 
inflammation tissue damage in arthritis. J Immunol. 2009;183(5):3383-3389.

10. Uderhardt S, et al. 12/15-lipoxygenase orchestrates the clearance of apoptotic cells maintains immunologic tolerance. Immunity. 2012;36(5):834-846.

11. Uderhardt S, Krönke G. 12/15-lipoxygenase during the regulation of inflammation, immunity, self-tolerance. J Mol Med (Berl). 2012;90(11):1247-1256.

12. Spanbroek R, et al. IL-4 determines eicosanoid formation in dendritic cells by down-regulation of 5-lipoxygenase up-regulation of 15-lipoxygenase 1 expression. Proc Natl Acad Sci U S A. 2001;98(9):5152-5157.

13. Shamshiev AT, Ampenberger F, Ernst B, Rohrer L, Marsland BJ, Kopf M. Dyslipidemia inhibits toll-like receptor-induced activation of CD8alpha-negative dendritic cells protective Th1 type immunity. J Exp Med. 2007;204(2):441-452.

14. Morgan AH, et al. Phosphatidylethanolamine-esterified eicosanoids in the mouse:tissue localization and inflammation-dependent formation in Th-2 disease. J Biol Chem. 2009;284(32):21185-21191.

15. Kadl A, et al. Identification of a novel macrophage phenotype that develops in response to atherogenic phospholipids via Nrf2. Circ Res. 2010;107(6):737-746.

16. Vignali DA, Kuchroo VK. IL-12 family cytokines:immunological playmakers. Nat Immunol. 2012;13(8):722-728.

17. Pierson E, Simmons SB, Castelli L, Goverman JM. Mechanisms regulating regional localization of inflammation during CNS autoimmunity. Immunol Rev. 2012;248(1):205-215.

18. Kuhn H. Structural basis for the positional specificity of lipoxygenases. Prostaglandins Other Lipid Mediat. 2000;62(3):255-270.

19. Blüml S, et al. Oxidized phospholipids negatively regulate dendritic cell maturation induced by TLRs and CD40. JImmunol. 2005;175(1):501-508.

20. Blüml S, et al. Epigenetic regulation of dendritic cell differentiation and function by oxidized phospholipids. Blood. 2009;114(27):5481-5489.

21. Asquith DL, Miller AM, Hueber AJ, Liew FY, Sattar N, McInnes IB. Apolipoprotein E-deficient mice are resistant to the development of collagen-induced arthritis. Arthritis Rheum. 2010;62(2):472-477.

22. Angeli V, et al. Dyslipidemia associated with ath- erosclerotic disease systemically alters dendritic cell mobilization. Immunity. 2004;21(4):561-574.

23. Cyrus $\mathrm{T}$, et al. Absence of $12 / 15$-lipoxygenase expression decreases lipid peroxidation atherogenesis in apolipoprotein e-deficient mice. Circulation. 2001;103(18):2277-2282.

24. Berliner JA, Subbanagounder G, Leitinger N, Watson AD, Vora D. Evidence for a role of phospholipid oxidation products in atherogenesis. Trends Cardiovasc Med. 2001;11(3-4):142-147.

25. Al-Huseini LM, et al. Nuclear factor-erythroid 2 (NF-E2) p45-related factor-2 (Nrf2) modulates dendritic cell immune function through regulation of p38 MAPK-cAMP-responsive element binding protein/activating transcription factor 1 signaling. JBiol Chem. 2013;288(31):22281-22288.

26. Aw Yeang HX, et al. Loss of transcription factor nuclear factor-erythroid 2 (NF-E2) p45related factor-2 (Nrf2) leads to dysregulation of immune functions, redox homeostasis, intracellular signaling in dendritic cells. J Biol Chem. 2012;287(13):10556-10564.

27. Rangasamy T, et al. Nuclear erythroid 2 p 45related factor 2 inhibits the maturation of murine dendritic cells by ragweed extract. Am J Respir Cell Mol Biol. 2010;43(3):276-285.

28. Williams MA, et al. Disruption of the transcription factor Nrf2 promotes pro-oxidative dendritic cells that stimulate Th2-like immunoresponsiveness upon activation by ambient particulate matter. Jimmunol. 2008;181(7):4545-4559.

29. Middleton MK, Rubinstein T, Puré E. Cellular and molecular mechanisms of the selective regulation of IL-12 production by 12/15-lipoxygenase. JImmunol. 2006;176(1):265-274.

30. Zhao L, et al. Selective interleukin-12 synthesis defect in 12/15-lipoxygenase-deficient macrophages associated with reduced atherosclerosis in a mouse model of familial hypercholesterolemia. J Biol Chem. 2002;277(38):35350-35356.

31. Sheibanie AF, Tadmori I, Jing H, Vassiliou E, Ganea D. Prostaglandin E2 induces IL-23 production in bone marrow-derived dendritic cells. FASEB J. 2004;18(11):1318-1320.

32. Emerson MR, LeVine SM. Experimental allergic encephalomyelitis is exacerbated in mice deficient for 12/15-lipoxygenase or 5-lipoxygenase. Brain Res. 2004;1021(1):140-145.

33. Johnson DA, Amirahmadi S, Ward C, Fabry Z, Johnson JA. The absence of the pro-antioxidant transcription factor Nrf2 exacerbates experimen- tal autoimmune encephalomyelitis. Toxicol Sci. 2010;114(2):237-246.

34. Pareek TK, et al. Triterpenoid modulation of IL-17 and Nrf-2 expression ameliorates neuroinflammation promotes remyelination in autoimmune encephalomyelitis. Sci Rep. 2011;1:201.

35. Bar-Or A, et al. Clinical efficacy of BG-12 (dimethyl fumarate) in patients with relapsing-remitting multiple sclerosis:subgroup analyses of the DEFINE study. JNeurol. 2013;260(9):2297-2305.

36. Gold R, et al. Placebo-controlled phase 3 study of oral BG-12 for relapsing multiple sclerosis. $N$ Engl JMed.2012;367(12):1098-1107.

37. Dobrian AD, Lieb DC, Cole BK, Taylor-Fishwick DA, Chakrabarti SK, Nadler JL. Functional and pathological roles of the 12- and 15-lipoxygenases. Prog Lipid Res. 2011;50(1):115-131.

38. Itoh K, et al. An Nrf2/small Maf heterodimer mediates the induction of phase II detoxifying enzyme genes through antioxidant response elements. Biochem Biophys Res Commun. 1997;236(2):313-322.

39. Zal T, Volkmann A, Stockinger B. Mechanisms of tolerance induction in major histocompatibility complex class II-restricted T cells specific for a blood-borne self-antigen. J Exp Med. 1994;180(6):2089-2099.

40. Lutz MB, et al. An advanced culture method for generating large quantities of highly pure dendritic cells from mouse bone marrow. J Immunol Methods. 1999;223(1):77-92.

41. Pfeiffer IA, et al. Leukoreduction system chambers are an efficient, valid, and economic source of functional monocyte-derived dendritic cells and lymphocytes. Immunobiology. 2013;218(11):1392-1401.

42. von Schlieffen E, et al. Multi-hit inhibition of circulating and cell-associated components of the toll-like receptor 4 pathway by oxidized phospholipids. Arterioscler Thromb Vasc Biol. 2009;29(3):356-362.

43. Watson AD, et al. Structural identification by mass spectrometry of oxidized phospholipids in minimally oxidized low density lipoprotein that induce monocyte/endothelial interactions and evidence for their presence in vivo. J Biol Chem. 1997;272(21):13597-13607.

44. Krönke G, et al. Oxidized phospholipids induce expression of human heme oxygenase-1 involving activation of cAMP-responsive element-binding protein. JBiol Chem. 2003;278(51):51006-51014. 